\title{
Dark energy equation of state and anthropic selection
}

\author{
Jaume Garriga \\ Departament de Física Fonamental, Universitat de Barcelona, Diagonal 647, 08028 Barcelona, Spain \\ and IFAE, Campus UAB, 08193 Bellaterra (Barcelona), Spain
}

Andrei Linde

Department of Physics, Stanford University, Stanford, California 94305-4060, USA

Alexander Vilenkin

Institute of Cosmology, Department of Physics and Astronomy, Tufts University, Medford, Massachusetts 02155, USA

(Received 15 October 2003; published 29 March 2004)

\begin{abstract}
We explore the possibility that the dark energy is due to a potential of a scalar field and that the magnitude and the slope of this potential in our part of the Universe are largely determined by anthropic selection effects. We find that, in some models, the most probable values of the slope are very small, implying that the dark energy density stays constant to very high accuracy throughout cosmological evolution. In other models, however, the most probable values of the slope are such that the slow roll condition is only marginally satisfied, leading to a recollapse of the local universe on a time scale comparable to the lifetime of the Sun. In the latter case, the effective equation of state varies appreciably with the redshift, leading to a number of testable predictions.
\end{abstract}

DOI: $10.1103 /$ PhysRevD.69.063521

PACS number(s): $98.80 . \mathrm{Cq}$

\section{INTRODUCTION}

It has long been suggested that both the old fine-tuning problem of the cosmological constant as well as the puzzle of the time coincidence may find a natural explanation through anthropic selection effects, in scenarios where the dark energy density $\rho_{D}$ is a random variable $[1-16]$. This possibility can be easily realized in the context of inflationary cosmology, where the local value of $\rho_{D}$ may be determined by stochastic quantum processes. These processes may lead to rather different values of $\rho_{D}$ in distant regions of the Universe, separated by length scales much larger than the present Hubble radius.

A simple implementation of this idea is obtained $[6,17]$ by assuming that the dark energy is due to a scalar field $\phi$ (different from the inflaton field) with a very flat potential $V(\phi)$, which has a simple zero at $\phi=\phi_{0}$ with a nonvanishing slope $s \equiv\left|V^{\prime}\left(\phi_{0}\right)\right|$ :

$$
V(\phi)=-s\left(\phi-\phi_{0}\right)+O\left[\left(\phi-\phi_{0}\right)^{2}\right],
$$

where we have assumed for definiteness that $V^{\prime}\left(\phi_{0}\right)<0$. All that is required is that the slow-roll conditions

$$
\left|V^{\prime}\right| \lesssim H_{0}^{2} M_{P}, \quad\left|V^{\prime \prime}\right| \lesssim H_{0}^{2}
$$

are satisfied for values of the potential in the relatively narrow range

$$
|V| \lesssim 10^{3} M_{P}^{2} H_{0}^{2} .
$$

Here $H_{0}$ is the present expansion rate and $M_{P}$ is the reduced Planck mass, and we are adopting the convention that any contributions to the vacuum energy (such as a true cosmological term) are included in the definition of $V(\phi)$. Larger values of $|V|$ are uninteresting, since they would severely interfere with structure formation and with the emergence of suitable observers. During inflation, the value of the scalar field $\phi$ is randomized by quantum fluctuations, and after inflation it stays almost frozen due to the flatness of the potential. Thus, the local value of the dark energy density $\rho_{D}$ $\approx V(\phi)$ will vary from place to place, but it will stay almost constant in time. In this situation, the probability for measuring a particular value of $\rho_{D}$ is determined by a combination of inflationary dynamics and anthropic selection effects. As we shall see in the next section, this approach to the cosmological constant problems shows remarkable agreement with observations, even with the crudest of assumptions.

The purpose of the present paper is to extend this analysis to scenarios where the slope $s$ of the potential is itself a random variable. Like $\rho_{D}$, the measured value of the slope could be determined by a combination of inflationary dynamics and anthropic selection effects. A very large slope would cause a big crunch much before any observers can develop. If the distribution which is obtained after inflation favors large values of $s$, then a value of the slope which marginally satisfies (2) could be the most probable one to observe [15,16,18]. Marginal slow-roll entails the consequence that the effective equation of state depends appreciably on redshift, $p_{D}=w_{s}(z) \rho_{D}$, through a function $w_{s}$ which contains a single parameter: the value of the slope $s$ in our region of the Universe. Thus, the equation of state (and its time evolution) may ultimately be determined by the condition that galaxy formation and the emergence of suitable observers is marginally allowed before the big crunch happens. Some observational signatures of models with a marginal slope have been discussed in $[15,18,19,21]$.

In Sec. II we review the case of variable $\rho_{D}$ at fixed $s$. In Sec. III we discuss two-field models of dark energy, where both $\rho_{D}$ and $s$ are random variables. Our conclusions are summarized in Sec. IV. 


\section{VARIABLE $\rho_{D}$}

\section{A. Prior distribution}

As mentioned in the Introduction, a theory with variable $\rho_{D}$ can be obtained from a scalar field with a very flat potential, as in Eq. (1). During inflation, the field $\phi$ undergoes a random walk of step size $\delta \phi \sim H$ for each time interval $\delta t \sim H^{-1}$, where $H$ is the expansion rate during inflation. The steps are taken independently on each horizon volume, and this leads to spatial variation of $\phi$. The potential is very flat, and appreciable spatial variation of $\rho_{D}$ after thermalization will only occur on scales much larger than the presently observable Universe.

In the limiting case when the potential is absolutely flat, the rate of expansion of the Universe does not depend on the value of the field $\phi$. Then, because of the Brownian motion of the field $\phi$ during eternal inflation, the field takes all possible values with equal probability. In other words, the volume distribution of the field $\phi$ at thermalization does not depend on the value of the field and takes the form

$$
d \mathcal{P}_{*} \propto d \phi
$$

When one takes into account that the potential is not entirely flat, the situation becomes more complicated. The probability distribution acquires some $\phi$ dependence, which may be sensitive to a particular choice of the measure of probability in an eternally inflating universe. This is a rather delicate issue, see, e.g. [23,24], but the final results may not be very sensitive to it because of the extreme flatness of the potentials suitable for the description of dark energy. It has been argued in [17] that for a particular choice of the measure, and provided that certain generic conditions are satisfied, the volume distribution of the field $\phi$ at thermalization preserves the simple form (4) in the narrow range of anthropic interest (3). We shall return to this issue in a bit more detail in the next section, where the case with several dark energy fields is considered. As we shall see, additional subtleties arise in that context which require further discussion. For the rest of this section, we shall assume that we are indeed in the situation where the flat distribution (4) is valid.

From the end of inflation until the present time, the field is heavily overdamped and remains almost frozen, giving a nearly time-independent contribution to $\rho_{D}$. Thus, the "prior" distribution for the dark energy density is given by

$$
d \mathcal{P}_{*} \propto \frac{d \rho_{D}}{\left|V^{\prime}(\phi)\right|} .
$$

Consider, for illustration, the simplest linear potential

$$
V(\phi)=\alpha \phi+\rho_{\Lambda}
$$

Here $\rho_{\Lambda}$ is a true cosmological constant. It is important that the cosmological constant can be removed from $V(\phi)$ by a simple redefinition of the field $\phi: \phi \rightarrow \phi-\rho_{\Lambda} / \alpha$. If the slope of the potential is sufficiently small (as in most of the models of dark energy),

$$
\alpha \lesssim 10^{-120} M_{p}^{3},
$$

then, according to Eq. (2), the field $\phi$ practically does not change during the last $10^{10}$ years, its kinetic energy is very small. Therefore at the present stage of the evolution of the Universe its total potential energy $V(\phi)$ acts nearly like a cosmological constant. Independently of $\rho_{\Lambda}$, this effective cosmological constant can take all possible values, including the presently observed extremely small value $\Lambda$ $\sim 10^{-120} M_{p}^{4}$.

Similarly, one may consider a model

$$
V(\phi)=\frac{1}{2} \mu^{2} \phi^{2}+\rho_{\Lambda} .
$$

Here $\rho_{\Lambda}$ is a true cosmological constant, and $\mu^{2} \rho_{\Lambda}<0$, so that, just as in the model (6), it is possible to have $\left|\rho_{D}\right|$ very small even if the constant $\left|\rho_{\Lambda}\right|$ is very large. Equation (2) leads to the condition [17]

$$
|\mu| \lesssim 10^{-120} M_{P}^{3}\left|\rho_{\Lambda}\right|^{-1 / 2} \text {. }
$$

Note that the bounds on $\alpha$ and $\mu$ do not correspond to a fine tuning, but just to a strong suppression. Possible mechanisms that could naturally account for such small values of parameters have been discussed in [17,18,20,25-27].

The potential vanishes at $\phi_{0}=-2 \rho_{\Lambda} / \mu^{2}$, and it can be easily checked that in the vicinity of this point we have

$$
\left|V^{\prime}(\phi)\right|=s\left[1+O\left(\rho_{D} / \rho_{\Lambda}\right)\right],
$$

where $s$ is the slope at $\phi=\phi_{0}$ and $\rho_{D} \approx V(\phi)$. Since the true cosmological constant is expected to be large in absolute value, we have $\rho_{D} \ll \rho_{\Lambda}$, and thus $V^{\prime}(\phi) \approx$ const in the range of interest, so the potential should be well approximated by a linear function (1). Substitution into (5) yields

$$
d \mathcal{P}_{*} \propto d \rho_{D}
$$

This means that all values of the dark energy density in the range (3) are equiprobable a priori.

A linear potential as a simple model for dark energy was first considered in [6]. Later it has been argued that this form of the potential is generic in the narrow anthropic range $[16,18,25]$.

It is instructive to compare these models to the more traditional models of dark energy, with potentials of the type $e^{-c Q}$ or $Q^{-\beta}$, where $Q$ is the quintessence field [28]. Generically, the potential of the quintessence field contains also a cosmological constant $V_{0}$, which, a priori, can be arbitrarily large and can have either sign. Thus, these models do not solve the cosmological constant problem. They also do not solve the coincidence problem, unless one fine tunes the parameters of the potential.

Since the quintessence potentials become asymptotically very flat, the scalar field $Q$ also experiences quantum fluctuations during inflation. Therefore, in these simple models, one should expect that the typical value of the quintessence field becomes indefinitely large in the process of eternal inflation. As a result, these models become completely indistinguishable from the theory with a simple cosmological constant $V_{0}$. 
One can solve all of these problems, including the cosmological constant problem, by adding the interaction of the field $Q$ with the curvature scalar, $\xi R Q^{2}$, and by multiplying the quintessence potential by $\phi^{n}$, where $\phi$ is a massless field [17]. In the simplest case $n=1$ the potential of the quintessence models [28] in the regime when $Q$ changes very slowly starts looking very similar to our simple linear model (1). Because of the new term $\sim \phi V_{0}$, one can solve the cosmological constant problem in the same way as in the model with the linear potential [6]. The modified quintessence model will have some features distinguishing it from the model (1), but overall it will be much more complicated.

\section{B. Full distribution}

The distribution $\mathcal{P}_{*}\left(\rho_{D}\right)$ cannot be interpreted directly as the probability for measuring a particular value of $\rho_{D}$. If $\left|\rho_{D}\right|$ is too large, so that it dominates prior to the galaxy formation epoch $t_{G} \sim 10^{10} \mathrm{yrs}$, then it will preclude the very existence of observers, and hence will never be measured. In order to implement this selection effect quantitatively, it seems reasonable to assume that we are typical observers in the ensemble of all observers in our thermalized region. The probability for measuring a particular value of $\rho_{D}$ can thus be taken to be proportional to the number of civilizations in the Universe which measure that value of $\rho_{D}$, and we have $[8,16]$

$$
d \mathcal{P}\left(\rho_{D}\right) \propto \int d M N_{c i v}\left(\rho_{D}, M\right) n\left(\rho_{D}, M\right) d \mathcal{P}_{*}\left(\rho_{D}\right) .
$$

Here, $n\left(\rho_{D}, M\right) d M$ is the number density of galaxies of mass in the interval $d M$ which will ever form in regions where the dark energy density takes the value $\rho_{D}$, and $N_{c i v}$ is the number of civilizations per galaxy. As a rough approximation we may assume that the integral is dominated by giant galaxies like the Milky Way, with mass $M \sim M_{G}$ $\sim 10^{12} M_{\odot}$, and that $N_{c i v}$ does not depend significantly on $\rho_{D}$. For $M \gtrsim M_{G}$, we may take $N_{c i v}$ to be proportional to the number of stars in the galaxy, or to the mass of the galaxy, $N_{c i v}(M) \propto M$. Thus, the probability for measuring a particular value of $\rho_{D}$ is proportional to the fraction of matter $f\left(M>M_{G}, \rho_{D}\right)$ which clusters in objects larger than $M_{G}$ in regions with this value of $\rho_{D}$. In the Press-Schechter approximation for determining the fraction of clustered matter in a $\Lambda \mathrm{CDM}$ model, and restricting attention to positive values of $\rho_{D}$, one finds $[10,16]$

$$
d \mathcal{P}\left(\rho_{D}\right) \propto f\left(M>M_{G}, \rho_{D}\right) d \rho_{D} \propto \operatorname{erfc}\left[.80 y^{1 / 3}\right] d y .
$$

Here, we have introduced the variable $y$ which is linearly related to the dark energy density

$$
y=\left[\frac{F\left(\Omega_{D 0} / \Omega_{m 0}\right)}{\sigma_{0}\left(M_{G}\right)}\right]^{3} \frac{\rho_{D}}{\rho_{D 0}},
$$

where $\sigma_{0}\left(M_{G}\right)$ is the present linearized density contrast on the galactic scale, $\Omega_{m 0}+\Omega_{D 0}=1, \Omega_{D 0}$ is the present value of $\Omega_{D}$ in our local region, and the function $F(x)$ is given by

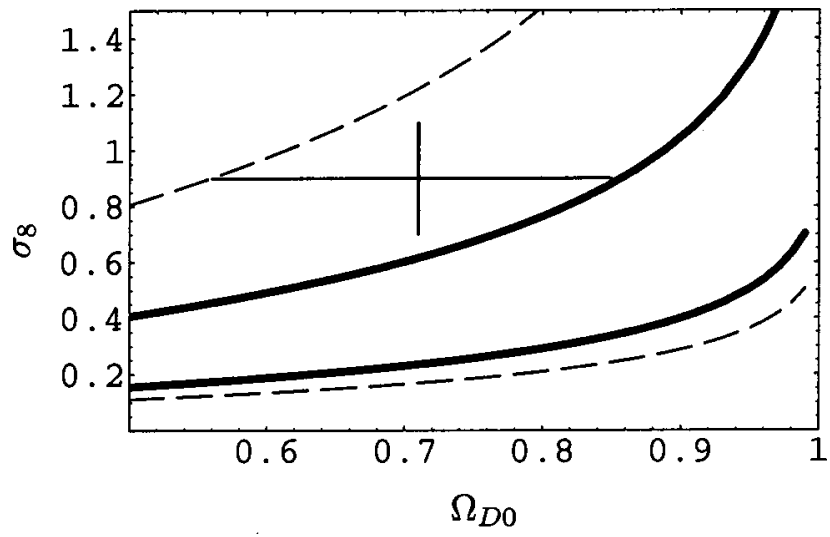

FIG. 1. Comparison of anthropic predictions with observations. The curves represent the boundaries of the $68 \%$ (solid) and $95 \%$ (dashed) confidence level regions predicted by the distribution (13). The cross represents the values inferred from Wilkinson Microwave Anisotropy Probe (WMAP) observations, with $2 \sigma$ error bars.

$$
F(x)=\frac{5}{6}\left(\frac{1+x}{x}\right)^{1 / 2} \int_{0}^{x} \frac{d w}{w^{1 / 6}(1+w)^{3 / 2}} .
$$

The distribution (13) predicts that $.33<y<6.0$ with $68 \%$ confidence and $.043<y<16$ at $95 \%$ confidence level. The observed value of $y$, given by

$$
y_{0}=\left[\frac{F\left(\Omega_{D 0} / \Omega_{m 0}\right)}{\sigma_{0}\left(M_{G}\right)}\right]^{3}
$$

is thus expected to fall into these intervals at $1 \sigma$ and $2 \sigma$ confidence levels, respectively. The boundaries of the intervals define curves in the $\Omega_{D 0^{-}}-\sigma_{0}$ plane. These curves are shown in Fig. 1, where instead of using the present density contrast $\sigma_{0}\left(M_{G}\right)$ on the galactic scale, we use the more familiar quantity $\sigma_{8}$. For a given value of $\Omega_{D 0}, \sigma_{8}$ can be obtained from $\sigma_{0}\left(M_{G}\right)$ if the cosmological parameters such as the spectral index of density perturbations $n$, the dimensionless Hubble constant $h$ and the baryon fraction $\Omega_{b}$ are known. For these parameters we have taken the central values given by WMAP, $n=.99, h=.72$, and $\Omega_{b}=.047$ [29]. Also shown in the same plot are the values inferred from WMAP for $\sigma_{8}$ and $\Omega_{D 0}$, which fall well within the anthropic predictions at the $95 \%$ confidence level. Given the simplicity of the assumptions which have been made, the agreement between predictions and observations seems quite remarkable.

For negative values of $\rho_{D}$, the structure formation proceeds as usual until the time $t_{D} \sim\left(G\left|\rho_{D}\right|\right)^{-1 / 2}$, when the matter density $\rho_{m}$ becomes comparable to $\left|\rho_{D}\right|$. At the moment when $\rho_{m}=\left|\rho_{D}\right|$, the universe stops its expansion and starts recontraction. The effect of a negative $\rho_{D}$ is in many ways similar to the effect of the slope, and to avoid duplication, we shall not discuss it here. Interested readers are referred to Refs. $[15,16]$, where it is argued that the probability for $\rho_{D}$ $<0$ is less or comparable to that of $\rho_{D}>0$. In the following discussion we shall focus on the positive values of $\rho_{D}$. 


\section{VARIABLE $\rho_{D}$ AND SLOPE}

In Ref. [16] two of the present authors considered several predictions of the anthropic approach to the cosmological constant problems, including the one we have just discussed in the previous section. Two other predictions were that the equation of state should be that of a cosmological constant, $w=-1$, and that the Universe would recollapse, but not before a trillion years. The latter predictions were based on the premise that, in a generic model, the slow-roll condition (2) is more likely to be satisfied by excess, by many orders of magnitude, rather than marginally. This seems clear in a model such as (8), where the slow-roll parameter is fixed, and a strong suppression of $\mu$ is required by the constraint (9). If the suppression is due to some symmetry, then it is natural to expect that this symmetry will make the potential flat by excess rather than marginally. Indeed, the symmetry only knows about microphysics, and a marginal value $|\mu|$ $\sim 10^{-120} M_{P}^{3}\left|\rho_{\Lambda}\right|^{-1 / 2}$ would itself represent a coincidence (or tuning) which requires separate explanation.

However, a marginal value of the slow-roll parameter may be obtained more naturally in models where the slope is itself a random variable, by invoking again anthropic selection effects. It was pointed out in [16] that if the prior distribution favors large $s$, then the most probable values of $s$ could be the marginal ones. However, the authors of [16] argued (as we will show, incorrectly) that the prior distribution after inflation necessarily favors small $s$, and concluded that the equation of state $w=-1$ should be expected in the general case. Dimopoulos and Thomas [18] suggested, on the contrary, that the prior should generally favor large $s$, but offered no explicit model to justify this claim. Here, we shall examine this issue in detail in the context of a specific model.

\section{A. Prior distribution}

A variable slope is easily obtained by considering a model where we have several fields $\varphi_{a}$ instead of just one, so that on the hypersurface $V\left(\varphi_{1}, \varphi_{2}, \ldots, \varphi_{n}\right)=0$ the gradient $s(\varphi) \equiv|\nabla V|$ depends on $\varphi_{a}$. During inflation, quantum fluctuations cause a random walk in field space which covers a distance $\left|\Delta \varphi_{a}\right| \sim H(H t)^{1 / 2}$ in time $t$, where $H$ is the inflationary expansion rate. If we start with some probability distribution $d \mathcal{P}_{*}\left(\varphi_{a}, t_{i}\right)$ at some initial time $t_{i}$, the random walk causes "diffusion" of probability in field space, which tends to flatten the distribution as inflation proceeds. Hence, if we neglect the effect of the fields $\varphi_{a}$ on the expansion of the universe, we should expect that the volume distribution at the time of thermalization is given by

$$
d \mathcal{P}_{*}\left(\varphi_{a}\right) \propto \prod_{a} d \varphi_{a}
$$

In general, however, the potential of the dark energy fields $V\left(\varphi_{a}\right)$ does contribute to the expansion, producing a nontrivial dependence of $\mathcal{P}_{*}$ on $\varphi_{a}$. Even though $V$ is very small compared with the inflationary energy scale (at least in the anthropically interesting range), it causes a "differential expansion" which may accumulate during many e-foldings, bi- asing the distribution towards values of the field where $V\left(\varphi_{a}\right)$ is larger. This is simply because the volume of regions where the potential is larger grows faster. This leads to a field-dependent distribution,

$$
d \mathcal{P}_{*}\left(\varphi_{a}\right)=F\left(\varphi_{a}\right) \prod_{a} d \varphi_{a}
$$

In a region of size $\Delta \varphi$ in the field space, the characteristic quantum diffusion time is of the order $\tau_{q} \sim(\Delta \varphi)^{2} / H^{3}$, and the time scale on which the differential expansion becomes important is $\tau_{d e} \sim(\Delta H)^{-1} \sim H M_{p}^{2} / \Delta V \sim H M_{p}^{2} /\left|\nabla_{\varphi} V\right| \Delta \varphi$. Diffusion will make the function $F\left(\varphi_{a}\right)$ very smooth (or nearly constant) on scales $\Delta \varphi$ smaller than the smearing scale

$$
\Delta \varphi_{\text {smear }} \sim H\left(\frac{H M_{p}^{2}}{\left|\nabla_{\varphi} V\right|}\right)^{1 / 3},
$$

which is obtained by setting $\tau_{d e} \sim \tau_{q}$ [30]. On larger scales, $F$ will generally have a nontrivial dependence on $\varphi_{a}$.

In the case we discussed in Sec. II, where there is a single dark energy field $\phi$, one can argue that since the anthropic range for $\rho_{D}$ is rather narrow, the corresponding range of $\phi$ is also limited, and may easily be smaller than the smearing range (18). This has been used $[16,17]$ in order to justify the use of the flat distribution (4) under certain generic conditions. However, this justification becomes less clear when we have several dark energy fields, since the anthropic range does not necessarily correspond to a small compact region in the field space, and $F$ can vary significantly along the noncompact directions.

Not much can be said about $F$ in general, since its form depends on the overall shape of the inflaton and dark energy potential. (For a given potential, and with additional assumptions about the measure, $F$ can in principle be calculated by solving a suitable Fokker-Planck equation in the formalism of stochastic inflation [22-24]). To simplify our subsequent discussion, here we shall restrict ourselves to the case where differential expansion is negligible in the field range of interest. This is achieved for instance through a potential of the form

$$
U\left(\psi, \varphi_{a}\right)=U(\psi)+f(\psi) V\left(\varphi_{a}\right),
$$

where $\psi$ is the inflaton and $\varphi_{a}$ are the dark energy fields. The function $f(\psi)$ is normalized to unity in the thermalized phase, so that the potential $V\left(\varphi_{a}\right)$ becomes the dark energy density. If $f(\psi)$ is sufficiently small in the range of $\psi$ corresponding to most of the inflationary phase, then the differential expansion can be neglected and the prior distribution will take the form (4).

The minimal number of fields required to account for variation of $\rho_{D}$ and $s$ is $n=2$. In this case, $s=\left(V_{, 1}^{2}+V_{, 2}^{2}\right)^{1 / 2}$ and

$$
d \mathcal{P}_{*} \propto d \varphi_{1} d \varphi_{2} \propto|J|^{-1} d \rho_{D} d s
$$

where the Jacobian $J$ is given by 


$$
J \equiv \frac{\partial(V, s)}{\partial\left(\varphi_{1}, \varphi_{2}\right)}=s^{-1} \epsilon_{a b} V_{, a} V_{, b c} V_{, c}
$$

and $V_{, a} \equiv \partial V / \partial \varphi_{a}$.

A simple example is given by

$$
V=a \phi+U(\chi)+\rho_{\Lambda},
$$

where $\phi_{a}=(\phi, \chi), a$ is a constant coefficient and $\rho_{\Lambda}$ is the cosmological constant." This model can be considered as a generalized combination of two models described by Eqs. (6) and (8). The cosmological constant $\rho_{\Lambda}$ can be removed from this potential by redefinition of the field $\phi$, just as in the model (6). Thus the probability to live in the Universe with an effective cosmological constant $\rho_{D}$ does not depend on the true cosmological constant $\rho_{\Lambda}$, so in the future we will drop it in the expression for $V$.

The properties of the corresponding probability distribution can be easily understood by expressing it in terms of the variables $\rho_{D}$ and $\chi$. This gives

$$
d \mathcal{P}_{*} \propto d \rho_{D} d \chi
$$

where we have used the Jacobian $\partial(V, \chi) / \partial(\phi, \chi)=\partial V / \partial \phi$ $=a=$ const. Thus, for a given interval of $\rho_{D}$, all values of $\chi$ are equally probable. If the range of $\chi$ is unbounded, then the character of the distribution is determined largely by the asymptotic behavior of $U(\chi)$. If $U^{\prime}(\chi)$ is a growing function of $\chi$, then at large $\chi$ the slope is $s \approx\left|U^{\prime}(\chi)\right|$, and the probability is dominated by large values of the slope. For example, in the simplest case $U(\chi) \propto \chi^{2}$ we have $s \propto \chi$, and all values of $s$ are equally probable (for sufficiently large $s$ ). Alternatively, if $U^{\prime}(\chi) \rightarrow 0$ at $|\chi| \rightarrow \infty$, then the value $s=a$ is favored.

For a potential of the form

$$
V=a \phi+\frac{b}{n} \chi^{n}
$$

where $a, b$ and $n$ are constants, the slope is given by

$$
s=\left(a^{2}+b^{2} \chi^{2(n-1)}\right)^{1 / 2},
$$

and the Jacobian (21) is

$$
J \propto \chi^{2 n-3 / s} \propto s^{-1}\left(s^{2}-a^{2}\right)^{(2 n-3) / 2(n-1)} .
$$

Note that this expression depends only on $s$, but not on $\rho_{D}$, and thus the distribution factorizes

$$
d \mathcal{P}_{*}=\mathcal{P}_{*}(s) d s d \rho_{D},
$$

with

$$
\mathcal{P}_{*}(s) \propto s\left(s^{2}-a^{2}\right)^{-(2 n-3) / 2(n-1)} .
$$

Then, away from a small range of $s$ near zero, the distribution (28) has a power-law form

$$
\mathcal{P}_{*}(s) \propto s^{-1} s^{1 /(n-1)} .
$$

These results are applicable for the investigation of the effective cosmological constant produced by the fields $\phi$ and $\chi$ if these fields satisfy the slow roll conditions (2). The slow roll conditions for the field $\phi$, just as in the model (6), require that $a \lesssim H_{0}^{2} \sim 10^{-120}$ in units $M_{p}=1$, see Eq. (7), and the same logic as above leads us to expect this condition to be satisfied by excess, that is, $a \ll 10^{-120}$. The slow roll conditions with respect to the field $\chi$ require a more detailed investigation. One can show that for $n>1$ and $b \gg \rho_{D}$ $\sim 10^{-120}$ the slow roll conditions for the field $\chi$ are satisfied at the present stage of the evolution of the Universe only if $U(\chi) \ll \rho_{D}$. This makes the field $\chi$ irrelevant and returns us back to the one-field case, where we expect $w=-1$. The situation is more interesting for $a, b \leqq \rho_{D} \sim 10^{-120}$. In this case the slow-roll conditions for the field $\chi$ are satisfied for $\chi^{n-1} \lesssim b^{-1} 10^{-120}$. At the upper bound of this region, the slow-roll conditions are only marginally satisfied, which leads to a substantial deviation of $w$ from -1 .

Different behaviors of $\mathcal{P}_{*}(s)$ can now be obtained with a suitable choice of the parameter $n$. For $n \rightarrow \infty, \mathcal{P}_{*}(s) \propto s^{-1}$, so all logarithmic intervals of $s$ are equally probable. (This distribution is also obtained if the power law $b \chi^{n}$ is replaced by an exponential function $e^{b \chi}$.) For $n>1$, the distribution favors large values of $s$, and if $n$ is chosen close to 1 , the probability growth towards large $s$ can be made arbitrarily fast. With $n>1$, the prior distribution is non-integrable at large $s$, but in the next section we shall see that an effective cutoff is introduced by the galactic density factor $n\left(\rho_{D}, M_{G}\right)$, so the full distribution (12) is integrable.

For readers who are concerned about the appearance of non-integrable distributions, even at an intermediate stage of the analysis, we note that the divergence comes from $\chi$ $\rightarrow \infty$ and does not occur in models where the potential has the form (24) only in a finite range of $\chi$. In fact, Eq. (24) with $n=2$ can be thought of as an expansion of a more general potential (22) in powers of $\chi$ near an extremum of $U(\chi)$.

Finally, for $n<1$, small values of $s$ are favored, and again, by choosing $n$ close to but below 1 , the distribution can be made arbitrarily steep. In this case, the distribution (28) is non-integrable at $s=a$. This singularity is not smoothed out by the galactic density factor, and the prediction of models with $n<1$ is that $s=a$ with probability $P$ $=1$. Since $a$ is expected to be very small, this prediction is observationally indistinguishable from $s=0$. Once again, the divergence can be cut off if the power-law form of the potential (24) applies only in a finite range of $\chi$.

\section{B. Galactic density}

The probability distribution for measuring given values of $\rho_{D}$ and $s$ is given by a straightforward generalization of Eq. (12),

$$
d \mathcal{P}\left(\rho_{D}, s\right) \propto \int d M N_{c i v}\left(\rho_{D}, s, M\right) n\left(\rho_{D}, s, M\right) d \mathcal{P}_{*}\left(\rho_{D}, s\right) .
$$

If the prior distribution $d \mathcal{P}_{*}$ favors small values of $s$, then 
we expect the fields $\varphi_{a}$ to be deep in the slow roll regime. In this case, $N_{c i v}$ and $n$ are practically independent of $s$, and we recover the results of Sec. II.

Suppose now that the prior favors large values of $s$. To simplify the discussion, we shall assume that the prior distribution has a factorized form (24) with a power-law distribution for $s$,

$$
\mathcal{P}_{*}(s) \propto s^{\beta},
$$

$\beta>-1$. For potentials of the form (24), $\beta$ is simply related to the power index $n, \beta=(2-n) /(n-1)$. (Note that $\beta=0$ for $n=2$, which is arguably the simplest choice.) As before, we shall assume that the integral in (30) is dominated by giant galaxies of mass $M \geqslant M_{G}$. Assuming also that for such galaxies

$$
N_{c i v}\left(\rho_{D}, s, M\right)=M \nu_{c i v}\left(\rho_{D}, s\right),
$$

where $\nu_{c i v}$ is the number of civilizations per unit mass, we have

$$
\begin{aligned}
d \mathcal{P}\left(\rho_{D}, s\right) \equiv & \mathcal{P}\left(\rho_{D}, s\right) d s d \rho_{D} \propto \nu_{c i v}\left(\rho_{D}, s\right) \\
& \times f\left(\rho_{D}, s, M>M_{G}\right) s^{\beta} d s d \rho_{D} .
\end{aligned}
$$

Here, $f$ is the fraction of matter which clusters in galaxies of size bigger than $M_{G}$. This fraction depends on the relative magnitude of three characteristic times: the galaxy formation time scale $t_{G} \sim 10^{10} \mathrm{yrs}$, the onset of vacuum-like dark energy domination $t_{D} \sim\left(G \rho_{D}\right)^{-1 / 2}$, and the recollapse time scale $t_{s}$ due to the slope of the potential (we shall estimate $t_{s}$ shortly). If $s$ is so small that $t_{s}$ is the largest of the three times, then the growth of density fluctuations effectively halts at $t_{D}$, and the comoving density of galaxies can be estimated as in Sec. II. No matter how small, the slope eventually causes the field to roll down to negative values of the potential, ending in a big crunch. In the contracting phase, the density fluctuations start growing again, and one might think that any galaxies that failed to form at $t<t_{D}$ would form then. However, "galaxies" that form at this epoch are likely to be very different from what we call "galaxies" now. At $t_{D}<t<t_{s}$, the dark energy density remains nearly constant, while matter density $\rho_{m}$ decreases exponentially with time, so at $t \sim t_{s}$ it is suppressed by an exponential factor. Moreover, in the course of the recollapse, the energy of the scalar field grows much faster than that of matter, and $\rho_{m} / \rho_{D}$ is further suppressed [31]. Hence, the contribution of nonrelativistic matter (like CDM or baryons) to the mass of bound objects formed during the recollapse is utterly negligible.

If $t_{D}$ is the largest of the three times, then the exponential suppression period is absent, but the rest of the above discussion still applies, and for $t \gg t_{s}$ the universe becomes scalar field dominated. Even at the onset of recollapse, $t \sim t_{s}$, galaxies as we know them may not be formed. In our part of the Universe, structure formation effectively stopped at $t$ $\sim t_{D}$, and the existing structures evolved more or less in isolation. This may account for the fact that disks of giant galaxies take their grand-design spiral form only relatively late, at $z \sim 0.3$. On the other hand, in a recollapsing universe the clustering hierarchy only speeds up at $t \gtrsim t_{s}$, and quiescent disks which may be necessary for the evolution of fragile creatures like ourselves may never be formed.

This discussion suggests that for $t_{D}<t_{s}$, the fraction of matter clustered in galaxies can be estimated as

$$
f\left(\rho_{D}, s, M>M_{G}\right) \sim f\left(\rho_{D}, M>M_{G}\right) \quad\left(t_{D}<t_{s}\right),
$$

where $f\left(\rho_{D}, M>M_{G}\right) \equiv f\left(r_{D}, 0, M>M_{G}\right)$ is the same which we used in the previous section, which can be read off from (13). In the opposite case, $t_{D}>t_{s}$, we expect that the density of habitable galaxies does not exceed the density of galacticsize halos that collapsed prior to $t_{s}$. To estimate this density, we shall need an estimate of $t_{s}$.

The field equation for $\varphi$ has the form

$$
\ddot{\varphi}+3(\dot{a} / a) \dot{\varphi}=s,
$$

where $a(t)$ is the scale factor. As long as the dark energy is subdominant, we have $a(t) \propto t^{2 / 3}$, and the solution of (34) is $\varphi=\varphi^{(0)}+\frac{1}{6} s t^{2}$, where we have imposed the initial condition $\dot{\varphi} \rightarrow 0$ at $t \rightarrow 0$. The dark energy density is then

$$
\rho_{D}(t)=\frac{1}{2} \dot{\varphi}^{2}-s \varphi=\rho_{D}{ }^{(0)}-\frac{1}{9} s^{2} t^{2} .
$$

Assuming first that $t_{s}<t_{D}$, we can disregard $\rho_{D}^{(0)}$, and the recollapse begins when the second term in (35) becomes comparable to the matter density $\rho_{m} \sim 1 / G t^{2}$,

$$
t_{s} \sim\left(M_{p} / s\right)^{1 / 2} .
$$

Alternatively, if the recollapse occurs after dark energy domination, then, for $\rho_{D}>0, a(t) \propto \exp \left(H_{D} t\right)$ with $H_{D}$ $\sim\left(G \rho_{D}\right)^{1 / 2}$. (Note that for $\rho_{D}<0$, the regime $t_{s} \gg t_{D}$ does not exist.) The solution of Eq. (34) is then $\varphi=\varphi^{(0)}$ $+\left(s / 3 H_{D}\right) t$, and the dark energy density is

$$
\rho_{D}(t)=\rho_{D}^{(0)}-\frac{s^{2}}{3 H_{D}} t .
$$

This equation applies as long as $\rho_{D}$ remains nearly constant. Recollapse begins when the second term in Eq. (37) becomes comparable to the first,

$$
t_{s} \sim \frac{H_{D} \rho_{D}}{s^{2}} \sim \frac{\rho_{D}^{3 / 2}}{M_{p} s^{2}} .
$$

The boundary between the two regimes is

$$
t_{s} \sim t_{D}: \quad s \sim \rho_{D} / M_{p} .
$$

Now, it follows from Eq. (36) that the matter density at $t_{s}$ is $\rho_{m}\left(t_{s}\right) \sim s M_{p}$, assuming $t_{s} \lesssim t_{D}$. This suggests that the fraction of matter in habitable galaxies in this regime is bounded by

$$
f\left(\rho_{D}, s, M>M_{G}\right) \leq f\left(s M_{p}, M>M_{G}\right) \quad\left(t_{D}>t_{s}\right) .
$$

In the estimates below, we shall use the value that saturates this inequality. 
We note finally that the dark energy density $\rho_{D}$ in Eqs. (30), (32) should be understood as the value of $\rho_{D}$ immediately after inflation, that is, the quantity denoted by $\rho_{D}^{(0)}$ in Eqs. (35), (37).

\section{The number of civilizations}

To estimate the dependence of the number of civilizations per unit mass $\nu_{c i v}$ on $\rho_{D}$ and $s$, we now have to consider the role of two other characteristic time scales: $t_{*} \sim(2-3)$ $\times 10^{10} \mathrm{yrs}$ - the time scale on which most of the main sequence stars believed to be suitable for life explode as red giants (see [11,32] for more discussion and references), and $t_{l}$ - the characteristic time needed for intelligent observers to evolve. $t_{I}$ is not likely to be much smaller than $t_{*}$, since then it is not clear why it took so long for intelligence to develop on Earth. Carter [33] has argued that $t_{I} \gg t_{*}$, since the coincidence $t_{I} \sim t_{*}$ is unlikely, considering that the evolution of life and the evolution of stars are governed by completely different processes. Note, however, that some seemingly unlikely coincidences may occur due to anthropic selection, $t_{G} \sim t_{D}$ being one example. Livio [32] has suggested a simple model illustrating how $t_{I} \sim t_{*}$ could arise. In any case, it seems reasonable to assume that

$$
t_{I} \gtrsim t_{*} .
$$

The time $t_{*}$ exceeds $t_{G}$ by only a factor $\sim 3$, but it will help to clarify the following discussion if we proceed as though $t_{*} \gg t_{G}$. This is justified in part by the fact that we will be comparing densities, which depend quadratically on time.

Observers can exist only in the time interval

$$
t_{G}<t<\min \left\{t_{s}, t_{*}\right\},
$$

and since according to Eq. (41) this interval is shorter than $t_{I}$, the number $\nu_{c i v}$ is suppressed by a certain factor. Assuming that the origin of intelligent life is due to a single and very infrequent random event which has some constant probability to occur per unit time, we have

$$
\nu_{c i v} \propto \min \left\{t_{s}, t_{*}\right\}-t_{G} .
$$

In practice, many steps are necessary for the development of intelligent life, some of them occurring much more frequently than others. Assuming that, out of the total number of steps, there are $k$ of them with typical frequencies smaller than $1 /\left[\min \left\{t_{s}, t_{*}\right\}-t_{G}\right]$, then Eq. (43) should be modified to

$$
\nu_{c i v} \propto\left(\min \left\{t_{s}, t_{*}\right\}-t_{G}\right)^{k} .
$$

Equation (44) assumes also that the steps which are needed to generate intelligence will produce the desired effect regardless of their time separation. This will not be the situation if there are relatively frequent catastrophes which occur at intervals shorter than $\left(\min \left\{t_{s}, t_{*}\right\}-t_{G}\right)$, and which are serious enough to erase memory of any previously achieved steps. In this case, a linear expression such as Eq. (43) is more appropriate.
Our current knowledge about the number of steps $k$ is very poor, and opinions differ quite vastly. Carter [33] has argued that the effective upper bound to the total time biological evolution can proceed on Earth, $t_{b}$, is likely to be in the range $t_{b}-t_{e} \lesssim t_{e} / k$, where $t_{e} \sim t_{*}$ is the actual time intelligent life has taken to develop on Earth. This formula reflects the fact that if many unlikely steps are necessary, then we are likely to have exhausted most of the available time before the emergence of intelligent life. If $k$ is large, Carter's formula seems to indicate that some catastrophe is awaiting right around the corner which will erase life from Earth within a time scale of order $t_{b}-t_{e} \ll t_{*}$. Carter rejected this possibility, and concluded that $k>2$ was not very likely. However, Barrow and Tipler [5] have argued that there is no reason to reject a relatively imminent doom caused, for instance, by some instability in the evolution of the Earth's atmosphere. This may render $t_{b}-t_{e}$ much shorter than $t_{*}$, and in this case there is no reason to expect that $k$ should be small. Cosmic doom of the type we discuss in this paper is yet another way of obtaining $t_{b}-t_{e} \ll t_{*}$, since $t_{b}<t_{s}$ and $t_{s}$ can in principle be smaller than $t_{*}$. Qualitatively, however, our results will not depend strongly on $k$, and for the rest of the discussion we shall just take $k=1$.

The right-hand side of Eq. (43) takes different forms, depending on the relative magnitude of $t_{s}, t_{D}$ and $t_{*}$. For $t_{s}$ $>t_{*}, \nu_{c i v}$ is independent of $s$ and $\rho_{D}$,

$$
\nu_{c i v} \sim \text { const } \quad\left(t_{s}>t_{*}\right) .
$$

For $t_{G} \ll t_{s}<t_{*}, \nu_{c i v} \propto t_{s}$, and using Eqs. (36),(38), we have

$$
\begin{array}{cc}
\nu_{c i v} \propto s^{-1 / 2} & \left(t_{s}<t_{*}, t_{D}\right), \\
\nu_{c i v} \propto \rho_{D}^{3 / 2} s^{-2} & \left(t_{D}<t_{s}<t_{*}\right) .
\end{array}
$$

The boundaries between the different regimes are given by Eq. (39) and by

$$
\begin{gathered}
t_{s} \sim t_{*} \quad\left(\rho_{D}<1 / G t_{*}^{2}\right): \quad s \sim M_{p} / t_{*}^{2}, \\
t_{s} \sim t_{*} \quad\left(\rho_{D}>1 / G t_{*}^{2}\right): \quad s \sim \rho_{D}^{3 / 4}\left(M_{p} t_{*}\right)^{-1 / 2} .
\end{gathered}
$$

The corresponding areas in the $s-\rho_{D}$ plane are sketched (not to scale) in Fig. 2. In our approximation, the factor $\nu_{c i v}$ vanishes for $t_{s}<t_{G}$. (In a more realistic treatment, the density of galaxies would not strictly vanish for small values of $t_{s}$. Galaxies would still be formed at high peaks of the density field, but their number density would be exponentially suppressed.) The boundary $t_{s} \sim t_{G}$ is homotetic to the boundary $t_{s} \sim t_{*}$ in the $s-\rho_{D}$ plane. For $s>\rho_{D} / M_{P}$ it simply corresponds to the vertical line at $s \sim M_{P} / t_{G}^{2}$, and for $s$ $<\rho_{D} / M_{P}$ it follows the curve

$$
s \sim \rho_{D}^{3 / 4}\left(M_{P} t_{G}\right)^{-1 / 2} .
$$

The behavior of $\nu_{c i v}$ as a function of $\rho_{D}$ and $s$ is illustrated in Fig. 3. In this and the following figures, $\rho_{D}$ is expressed in units of $\rho_{G} \equiv \rho_{m}\left(t_{G}\right)=(4 / 3) M_{p}^{2} t_{G}^{-2}$, where $t_{G}=t_{r e c} \sigma_{r e c}^{-3 / 2}$. Here, $\sigma_{r e c}$ is the density contrast on the galactic scale at the time of recombination. Roughly speaking, $\rho_{G}$ is the matter 


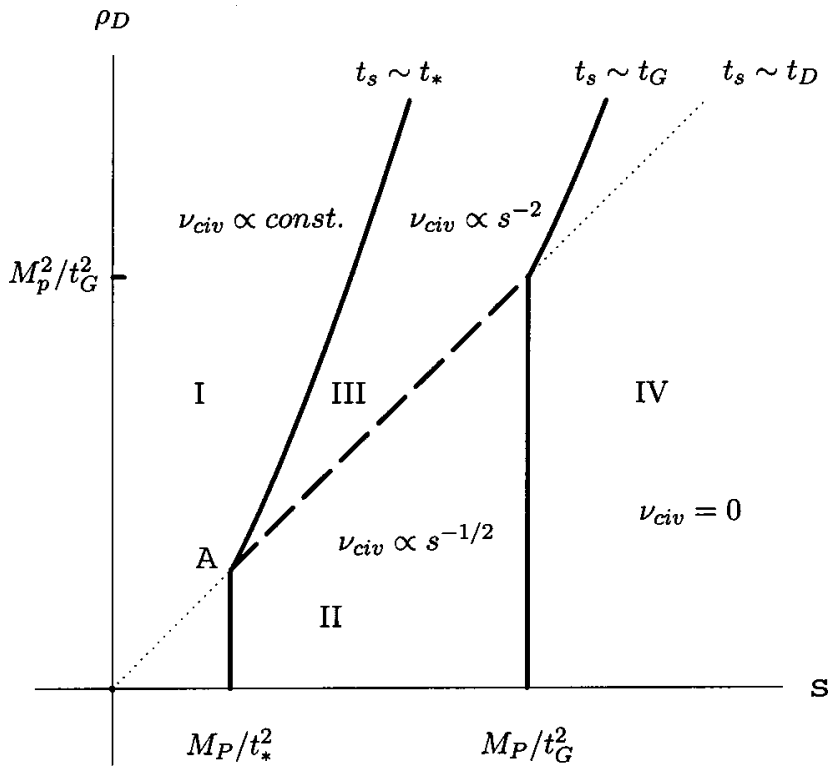

FIG. 2. Regions in the $s-\rho_{D}$ plane, illustrating the different behaviors of $\nu_{c i v}$ with $s$.

density at the time when the galaxies form, since $t_{G}$ is the time it takes for the linearized density contrast to become equal to 1 in the absence of a dark energy component. With these definitions, the variable $y$ which we used in Sec. II is the same as $\rho_{D} / \rho_{G}$.

\section{Full distribution}

We can now outline the general features of the full distribution (32). The effect of the galactic density factor $n\left(\rho_{D}, s, M_{G}\right)$ is, roughly, to cut off the distribution outside the square region $\rho_{D} \lesssim 1 / G t_{G}^{2}, s \lesssim M_{p} / t_{G}^{2}$. (These boundaries correspond to $t_{D} \sim t_{G}$ and $t_{s} \sim t_{G}$, respectively.) The fall-off, however, is rather mild, and $n\left(\rho_{D}, s, M_{G}\right)$ extends significantly outside the square. The cutoff is sharper in the $s$ direction, due to the rapid decline of $n_{c i v}$ with $s$. In region I $\mathcal{P} \propto S^{\beta}$, in region II $\mathcal{P} \propto s^{\beta-(1 / 2)}$, in region III $\mathcal{P} \propto s^{\beta-2}$, and in

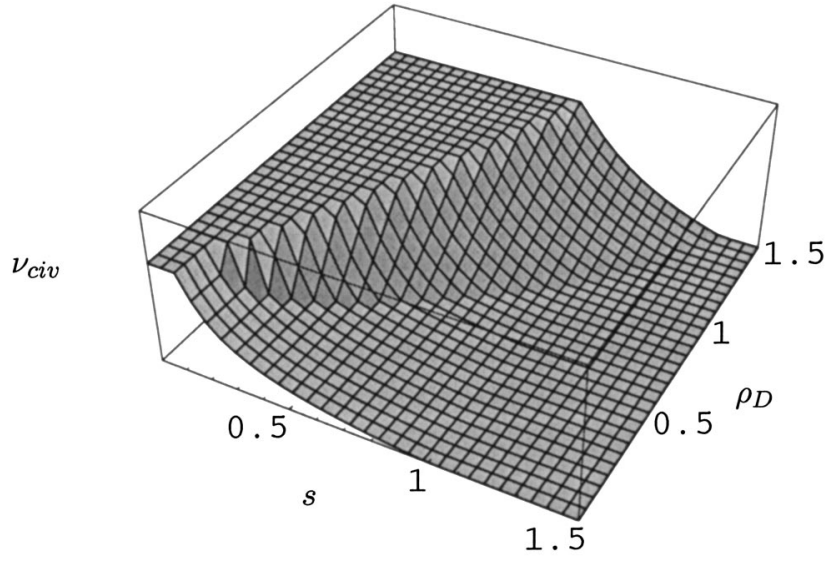

FIG. 3. Sketch of $\nu_{c i v}$ as a function of $s$ and $\rho_{D}$. For definiteness, we have used $t_{*}=3 t_{G}$. The slope $s$ is in units of $\rho_{G} / M_{p}$, whereas $\rho_{D}$ is in units of $\rho_{G}$.

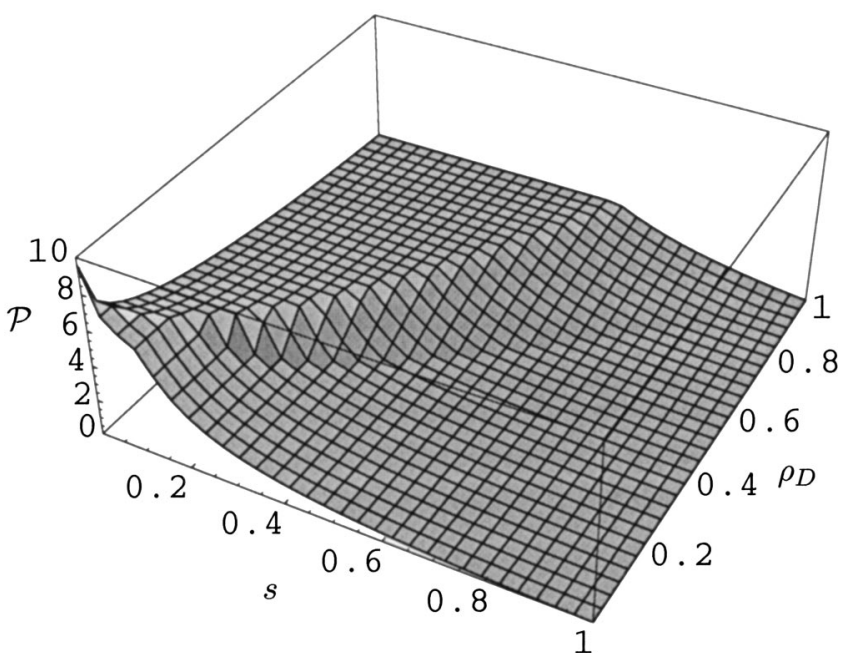

FIG. 4. The distribution $\mathcal{P}\left(s, \rho_{D}\right)$ as a function of $s$ and $\rho_{D}$, for $\beta=0$ [which corresponds to $n=2$ for a dark energy potential of the form given in Eq. (24)]. As in Fig. 3, $\rho_{D}$ is in units of $\rho_{G}$. The variable $y$ which we used in Sec. II is the same as $\rho_{D} / \rho_{G}$.

region IV $\mathcal{P} \approx 0$. The distribution $\mathcal{P}$ for $\beta=0[n=2$ in Eq. (24)] is illustrated in Fig. 4. The "logarithmic" distribution $s \rho_{D} \mathcal{P}\left(\rho_{D}, s\right)$ is illustrated in Figs. 5 and 6, for $\beta=0$ and $\beta$ $=2$, respectively.

For $-1 / 2<\beta<1$, the distribution $s \rho_{D} \mathcal{P}\left(\rho_{D}, s\right)$ is peaked along the line separating regions I and III (which corresponds to $t_{s} \sim t_{*}$ ). As we cross this "mountain range," moving towards larger values of $s$, the probability function drops in region III. The range terminates at point $A$ where regions I-III meet. For $-1<\beta<-1 / 2$, the range continues beyond point $A$ along the boundary between regions I and II.

The probability distribution for $\rho_{D}$ can be obtained by integrating over $s$,

$$
\mathcal{P}\left(\rho_{D}\right)=\int \mathcal{P}\left(\rho_{D}, s\right) d s .
$$

The character of this distribution depends on the value of $\beta$.

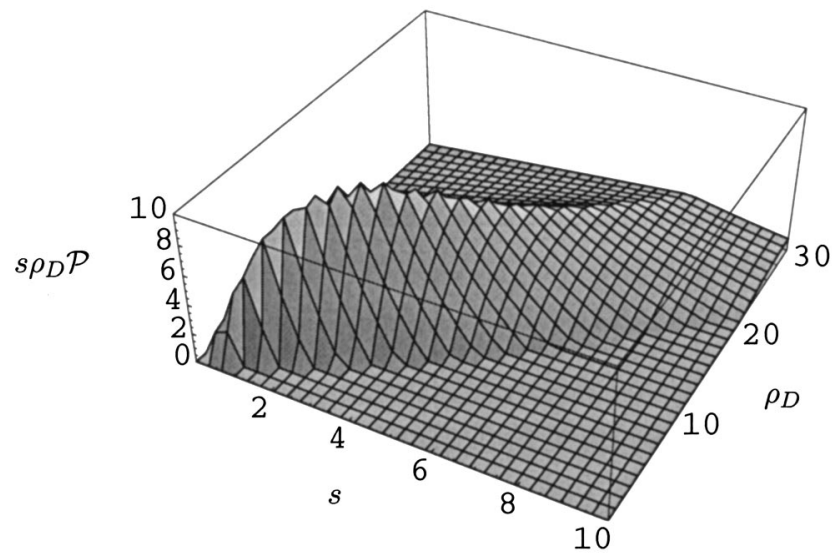

FIG. 5. The distribution $s \rho_{D} \mathcal{P}\left(s, \rho_{D}\right)$ as a function of $s$ and $\rho_{D}$, for $\beta=0$ (i.e. $n=2$ ). Same conventions as in Fig. 4 . 


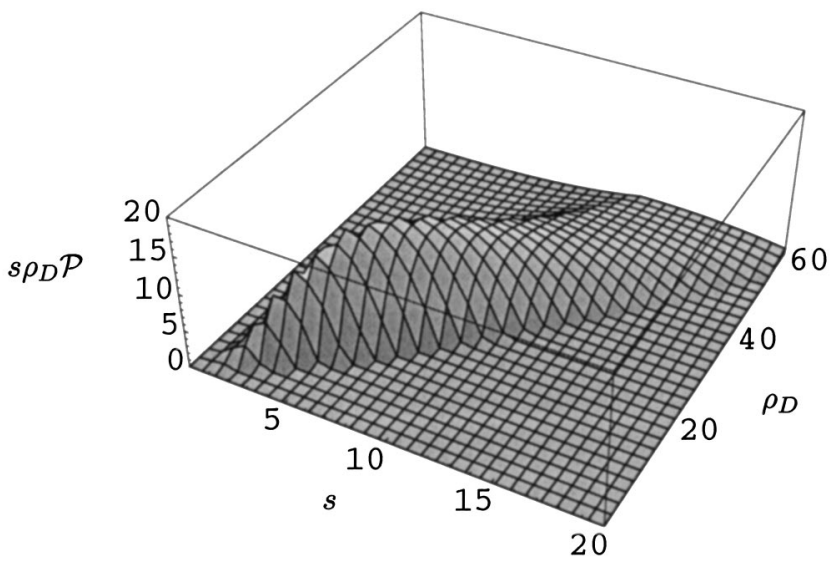

FIG. 6. The distribution $s \rho_{D} \mathcal{P}\left(s, \rho_{D}\right)$ for $\beta=2$ (i.e. $n=4 / 3$ ). The rest of parameter values and conventions are as in Figs. 4 and 5 .

For $\beta \approx-1$ or smaller, the prior distribution is peaked on a very narrow strip near $s=0$ in the $s-\rho_{D}$ plane. This strip is well within the flat plateau in $\nu_{c i v}$ at low $s$ (see Fig. 3). In this case, integration over $s$ just produces a constant factor independent of $\rho_{D}$, and the posterior distribution coincides with the distribution $\mathcal{P}\left(\rho_{D}\right)$ which is obtained for a true cosmological constant $(\mathrm{s}=0)$, given in (13). For $\beta$ in the range $-1<\beta \leqq 1$, but not too close to -1 , higher values of $s$ come into play. As shown in Fig. 3, the plateau in $\nu_{c i v}$ is broader in the $s$ direction at high $\rho_{D}$, and this feature is inherited by the function $\mathcal{P}\left(s, \rho_{D}\right)$ (shown in Fig. 4 for $\beta$ $=0)$. Thus, the integration over $s$ biases the distribution (51) towards larger $\rho_{D}$, relative to the case of a true cosmological constant. This effect gets stronger as $\beta$ increases.

Finally, for $\beta \gg 1$, the distribution is pushed to the largest possible values of $s$. For $\beta=2$, the distribution $s \rho_{D} \mathcal{P}\left(s, \rho_{D}\right)$ is plotted in Fig. 6. The probability is concentrated between the curves $t_{s} \sim t_{*}$ and $t_{s} \sim t_{G}$. If the value of $\beta$ is further increased, the distribution gets packed more towards $t_{s}$ $\sim t_{G}$, corresponding to Eq. (50). Larger $\beta$ means that larger $s$ is favored by the prior distribution, and from Eq. (50), this means that the posterior distribution is peaked at even larger $\rho_{D}$. The logarithmic distribution $\rho_{D} \mathcal{P}\left(\rho_{D}\right)$ is shown in Fig. 7 , for different values of $\beta$, ranging from -1 to 2 . The peak is indeed found to shift to larger values of $\rho_{D}$ as we increase $\beta$.

Figure 8 shows the $1-\sigma$ and $2-\sigma$ bounds on the variable $y=\rho_{D} / \rho_{G}$ as predicted by Eq. (51), as a function of the parameter $\beta$ in the prior distribution. The central value inferred from WMAP observations is $y_{0} \approx 0.1$, which for $\beta>$ -.5 lies outside the $2-\sigma$ confidence level region. However, two things should be noted before jumping to conclusions. First of all, there is a large uncertainty in the measured value of $y$ in our region of the universe. For instance, assuming the WMAP central values for the spectral index $n \approx .99$, the dimensionless Hubble constant $h \approx .72$ and the baryon fraction $\Omega_{b} \approx .047$, and taking into account the $2-\sigma$ error bars for $\sigma_{8}$ and $\Omega_{D}$ (depicted as a cross in Fig. 1), we find from Eq. (15) that the observed value of $y$ lies in the range $.04<y_{0}$

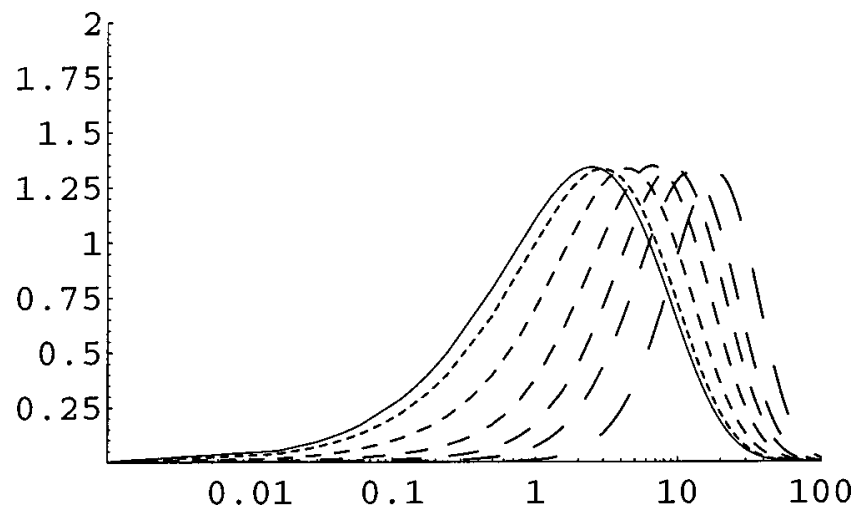

FIG. 7. The distribution $\rho_{D} \mathcal{P}\left(\rho_{D}\right)$ as a function of $\rho_{D} / \rho_{G}$ for different values of $\beta$. The solid line represents the standard case where $\rho_{D}$ is a true cosmological constant. This is recovered in the case that $\beta=-1$, since in this case the prior distribution has a non-integrable peak at $s=0$, where the slope of the potential vanishes. Also plotted are the cases $\beta=-.9,-.5,0, .5,1,2$ (dashed lines, where higher $\beta$ corresponds to longer dashes). The peak of the distribution shifts to higher $\rho_{D}$ as $\beta$ is increased.

$<$.48. Second, we should take into account that the predictions represented in Figs. 1 through 8 refer to the value of the dark energy density $\rho_{D}$ at some very early time, when the scalar field is still frozen by the cosmic expansion. For values of $s \gtrsim \rho_{G} / M_{P}$, the initial value of $\rho_{D}$ may be larger than the value at the present time by a sizable fraction. For instance, if the slope $s$ is such that the kinetic and potential energies of the dark energy field are approximately equal today (which may be considered a rather extreme case, although still marginally consistent with observations [19]) one finds that the dark energy density at very early times had to be larger by roughly a factor of 2 . Hence, the initial value of $\rho_{D} / \rho_{G}$ in our region of the Universe may well have been anywhere in the range $.04 \lesssim y \lessgtr 1$. The upper bound in this range is compatible with a value of $\beta \leqq 1$ (but not much higher) at the $2-\sigma$ level.

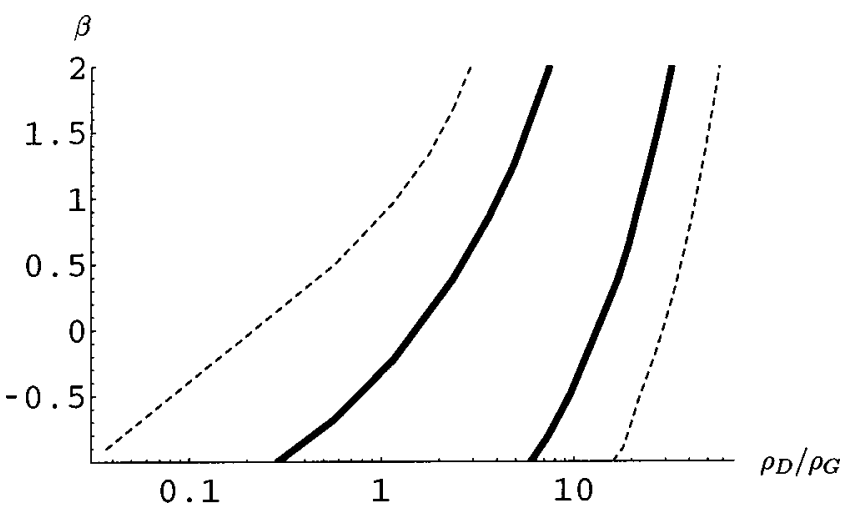

FIG. 8. 1- $\sigma$ (solid) and 2- $\sigma$ (dashed) bounds on the value of the variable $y=\rho_{D} / \rho_{G}$, as predicted by Eq. (51), as a function of the parameter $\beta$ in the prior distribution. Like in the previous figures, $\rho_{D}$ is the dark energy density at very early times, and $\rho_{G}$ is the matter density at the time of galaxy formation. The central value inferred from WMAP observations is $y \approx 0.1$, assuming that the dark energy density has remained approximately constant through cosmological evolution up to the present time. 
Given the rough nature of our model and the uncertainties in the data, our conclusions must be regarded as very preliminary. Nevertheless, our results do suggest that low values of $\beta \leq-0.5$ should give a better agreement with the data. This leaves two possibilities: either $\beta<-1$, in which case the probability is sharply peaked at $s \approx 0$, and all predictions of Ref. [16] for models with a fixed slope remain in force, or $-1<\beta \leqq 1$, and then the recollapse is most likely to occur at $t_{s} \sim t_{*}$. Since $t_{*}$ is comparable to the present age of the Universe, we can already expect to observe the signs of the slowdown of the cosmic expansion.

\section{CONCLUSIONS}

The possibility that the smallness of the observed effective cosmological constant, as well as the puzzle of the time coincidence, may be attributed to anthropic selection effects is rather tantalizing. To implement this idea, one assumes that $\rho_{D}$ is a random variable which takes different values in different parts of the universe (this could be due to stochastic quantum processes which took place during inflation). Observers cannot live in regions where $\rho_{D}$ is too large, since galaxies cannot form there, so we should not be surprised at the smallness of the observed $\rho_{D}$. Also, a simple analysis suggests that most observers will find themselves in regions which marginally allow the formation of suitable structures (e.g. galaxies of the type where observers are most likely to emerge). This would explain the time coincidence.

A pressing question regarding this scenario is whether it is possible at all to check its validity. In Ref. [16], two of the present authors ventured a few generic predictions of the anthropic approach to the cosmological constant problems. In particular, following up on the work of Martel, Shapiro and Weinberg [10], it was argued that the variable $y_{0}$ defined in Eq. (15) should be $y_{0}>.07$ with $95 \%$ probability. Assuming a cosmic background explorer normalized scale invariant spectrum of density perturbations, together with existing estimates for the baryon density, the anthropic argument suggested that the vacuum energy density parameter should be somewhat larger than $\Omega_{D}=.7$, or that the dimensionless Hubble rate should be somewhat smaller than $h=.7$. In Sec. II we have updated the comparison of predictions with observations by using the cosmological parameters as obtained from WMAP. Figure 1 shows confidence level plots for $\Omega_{D}$ and $\sigma_{8}$, corresponding to the $1-\sigma$ and $2-\sigma$ anthropic predictions, together with the values inferred from WMAP.

The agreement with current data is rather encouraging, and it will be interesting to see how it evolves as the level of precision increases. Note that the confidence level regions in Fig. 1 are rather broad. This corresponds to a genuine large variance in the cosmic distribution of $\rho_{D}$. Hence, one may be led to the conclusion that future observations will not bring much excitement, since the overall picture will remain qualitatively the same even if the observational error bars shrink by a large factor. Nevertheless, we should recall that a number of assumptions went into Fig. 1. For instance, we assumed that the spectral index for scalar fluctuations, the Hubble constant and the baryon fraction are given by the WMAP central values. We have also used $w \approx-1$ for the parameter in the dark energy equation of state. If the values of these parameters turn out to be different, the curves in Fig. 1 may shift significantly, putting some pressure on the anthropic explanation.

Also, we must consider the fact that we are quite ignorant about the conditions which are needed for the emergence of observers, an obvious drawback of the anthropic approach. However, if we can encode some of this ignorance in a few unknown physical parameters, we are in a position where we can predict something about the values of such parameters. By comparing the theoretical anthropic predictions with observations, we can find best fit estimates for the parameters, which may hopefully be confirmed some day by independent means. We have assumed throughout this paper that observers emerge predominantly in giant galaxies such as the Milky Way. This may be a reasonable assumption, but it is not an established fact by any means. Had we assumed that observers emerge predominantly in smaller galaxies, which form earlier on, the agreement with the data would become much worse. This reasoning was used in Ref. [16] to argue that the conditions for observers to emerge will be found predominantly in giant galaxies which complete their formation at redshift of order $z \sim 1$, but not much higher. This prediction seems hard to check at present, but hopefully much more will be known in the not so distant future about the properties of galaxies to confirm it or dispel it.

In this paper we have considered models where the dark energy is due to the potential energy of several scalar fields. In the case of a single field, one assumes that the potential has a simple zero at $\phi=\phi_{0}$ with nonvanishing slope $s$ (observers necessarily measure field values close to $\phi_{0}$, due to anthropic selection). If the slope is such that the slow-roll condition (2) is satisfied by excess, then the equation of state will be indistinguishable from that of a true cosmological constant. But if (2) is satisfied only marginally, then there will be substantial evolution of the equation of state parameter $w(z)$ with redshift. In models where the dark energy field has several components, both the dark energy density $\rho_{D}$ and the slope $s$ of the potential become random variables which take different values in distant regions of the Universe (separated by distances larger than the present Hubble radius). The function $w(z)$ (which is entirely determined by $\rho_{D}$ and $s$ ) will therefore be different in each one of these regions.

It was argued in Ref. [16] that in the case of a single dark energy field, the slow roll conditions (2) were likely to be satisfied by excess, by many orders of magnitude, rather than marginally. This leads to the predictions that the equation of state of dark energy is $p_{D}=-\rho_{D}$ with very high accuracy, and that the local universe will re-collapse, but not before another trillion years. It was also claimed that in generic two field models one should expect that small slopes would be favored by the prior distribution, leading to the same predictions as in the case of a single field. However, the latter conclusion was based on an incorrect analysis of the prior distribution, which we have amended in the present paper.

The prior distribution for the fields at the moment of thermalization can be obtained in principle from the inflationary dynamics. In the case of a single field, the anthropically al- 
lowed range (3) corresponds to a rather limited region in field space, and one can argue (under rather mild assumptions) that the prior distribution of the field will be almost flat within that range $[17,25]$. On the other hand, in the case where the dark energy potential involves several fields, the range (3) may correspond to a non-compact region in field space, and the prior distribution may be slowly varying in the non-compact directions. In this situation, the determination of the prior distribution from first principles is technically far more involved (and may require in general some further assumptions about the choice of the measure in an eternally inflating universe). Nevertheless, as argued in Sec. III, a flat distribution in field space can still be expected provided that the dark energy potential is sufficiently flat during inflation, so that its effect on the expansion can be completely neglected. In this situation, we have shown that there is a class of models where the prior distribution favors small slopes (in which case the conclusions of [16] hold) but there is an equally broad class of models where large values of the slope are favored a priori.

The measured value of $s$ is restricted by anthropic considerations, since if it is too large, the local region of the universe re-collapses before any observers have time to emerge. In Sec. III we have attempted to quantify this selection effect, and we have obtained posterior probability distributions for $\rho_{D}$ and $s$. The problem of estimating the abundance of observers in regions with given values of $\rho_{D}$ and $s$ has been split into two parts. In Sec. III B we have discussed how the abundance of suitable galaxies is determined as a function of $\rho_{D}$ and $s$, and in Sec. III C, we have analyzed how the number of civilizations may depend on these parameters. There is of course much room for improvement in these estimates, but even at the rough level at which they stand, they do illustrate the fact that a posterior distribution which favors a marginal slope can easily be obtained in models where the prior favors a large slope. In this case we find that the Universe is likely to turn around into contraction on a time scale comparable to the lifetime of the Sun. This is a quite exciting prospect since it may lead to a potentially observable timedependent equation of state $[15,18,19,21]$.

We finally comment on the string theory motivated picture of a "discretuum" of flux compactifications with different values of $\rho_{D}$ [13]. Recent work indicates that string theory does admit vacua with positive $\rho_{D}$ [34] and that the corresponding spectrum of $\rho_{D}$ may be rather dense [35], suggesting the possibility of anthropic selection $[13,36,37]$. We note, however, that a dense spectrum of possible values for $\rho_{D}$ is only a necessary, but not a sufficient condition for explaining the value we actually observe. The probability distribution $\mathcal{P}\left(\rho_{D}\right)$ depends on the prior distribution $\mathcal{P}_{*}\left(\rho_{D}\right)$, and in order to obtain reasonable agreement with observations, the prior should not be too different from the flat distribution (4). However, nearby values of $\rho_{D}$ in the discretuum picture correspond to very different values of the fluxes. The parts of the Universe with different values of $\rho_{D}$ will have very different evolution histories, and one might expect that their probabilities will also be rather different. The arguments we gave in Secs. I and III A for a flat prior distribution do not apply to this case. Calculation of probabilities in the discretuum remains an important problem for future research.

\section{ACKNOWLEDGMENTS}

The work by A.L. was supported by NSF grant PHY0244728 and by the Templeton Foundation Grant No. 938COS273. The work by J.G. was supported by CICYT Research Projects FPA2002-3598, FPA2002-00748, and DURSI 2001-SGR-0061. The work by A.V. was supported by the National Science Foundation.
[1] P.C. Davies and S.D. Unwin, Phys. Lett. 98B, 274 (1981).

[2] A.D. Linde, Rep. Prog. Phys. 47, 925 (1984).

[3] A.D. Sakharov, Zh. Éksp. Teor. Fiz. 87, 375 (1984) [Sov. Phys. JETP 60, 214 (1984)].

[4] T. Banks, Nucl. Phys. B249, 332 (1985).

[5] J.D. Barrow and F.J. Tipler, The Anthropic Cosmological Principle (Clarendon Press, Oxford, 1986).

[6] A.D. Linde, in 300 Years of Gravitation, edited by S.W. Hawking and W. Israel (Cambridge University Press, Cambridge, 1987).

[7] S. Weinberg, Phys. Rev. Lett. 59, 2607 (1987).

[8] A. Vilenkin, Phys. Rev. Lett. 74, 846 (1995).

[9] G. Efstathiou, Mon. Not. R. Astron. Soc. 274, L73 (1995).

[10] H. Martel, P.R. Shapiro, and S. Weinberg, Astrophys. J. 492, 29 (1998).

[11] J. Garriga, M. Livio, and A. Vilenkin, Phys. Rev. D 61, 023503 (2000).

[12] S.A. Bludman, Nucl. Phys. A663, 865 (2000).

[13] R. Bousso and J. Polchinski, J. High Energy Phys. 06, 006 (2000); J.L. Feng, J. March-Russell, S. Sethi, and F. Wilczek, Nucl. Phys. B602, 307 (2001).
[14] T. Banks, M. Dine, and L. Motl, J. High Energy Phys. 01, 031 (2001).

[15] R. Kallosh, A. Linde, S. Prokushkin, and M. Shmakova, Phys. Rev. D 66, 123503 (2002); R. Kallosh and A. Linde, ibid. 67, 023510 (2003).

[16] J. Garriga and A. Vilenkin, Phys. Rev. D 67, 043503 (2003).

[17] J. Garriga and A. Vilenkin, Phys. Rev. D 61, 083502 (2000).

[18] S. Dimopoulos and S. Thomas, Phys. Lett. B 573, 13 (2003).

[19] R. Kallosh, J. Kratochvil, A. Linde, E.V. Linder, and M. Shmakova, J. Cosmol. Astropart. Phys. 10, 015 (2003).

[20] R. Kallosh, A.D. Linde, D.A. Linde, and L. Susskind, Phys. Rev. D 52, 912 (1995).

[21] J. Garriga, L. Pogosian, and T. Vachaspati (in preparation).

[22] A. Vilenkin, Phys. Rev. D 27, 2848 (1983); A.A. Starobinsky, in Current Topics in Field Theory, Quantum Gravity and Strings, edited by H. Vega and N. Sanchez (Springer, Heidelberg, 1996).

[23] A.D. Linde, D.A. Linde, and A. Mezhlumian, Phys. Rev. D 49, 1783 (1994); J. Garcia-Bellido and A.D. Linde, ibid. 51, 429 (1995); A.D. Linde and A. Mezhlumian, ibid. 53, 4267 (1996). 
[24] A. Vilenkin, Phys. Rev. Lett. 81, 5501 (1998); V. Vanchurin, A. Vilenkin, and S. Winitzki, Phys. Rev. D 61, 083507 (2000); J. Garriga and A. Vilenkin, ibid. 64, 023507 (2001).

[25] S. Weinberg, Phys. Rev. D 61, 103505 (2000).

[26] J.F. Donoghue, J. High Energy Phys. 08, 022 (2000).

[27] G.R. Dvali and A. Vilenkin, Phys. Rev. D 64, 063509 (2001).

[28] C. Wetterich, Nucl. Phys. B302, 668 (1988); P.G. Ferreira and M. Joyce, Phys. Rev. D 58, 023503 (1998); B. Ratra and P.J. Peebles, ibid. 37, 3406 (1988); I. Zlatev, L.M. Wang, and P.J. Steinhardt, Phys. Rev. Lett. 82, 896 (1999).

[29] D.N. Spergel et al., Astrophys. J., Suppl. Ser. 148, 175 (2003).

[30] For a more detailed discussion of the relevant timescales, see Vanchurin et al. in [24].
[31] A general analysis of models with negative potentials has been given in G.N. Felder, A.V. Frolov, L. Kofman, and A.V. Linde, Phys. Rev. D 66, 023507 (2002).

[32] M. Livio, Astrophys. J. 511, 429 (1999).

[33] B. Carter, Philos. Trans. R. Soc. London 310, 347 (1983).

[34] S. Kachru, R. Kallosh, A. Linde, and S.P. Trivedi, Phys. Rev. D 68, 046005 (2003); C.P. Burgess, R. Kallosh, and F. Quevedo, J. High Energy Phys. 10, 056 (2003).

[35] M.R. Douglas, J. High Energy Phys. 05, 046 (2003); S. Ashok and M.R. Douglas, "Counting flux vacua," hep-th/0307049.

[36] L. Susskind, "The anthropic landscape of string theory," hep-th/0302219.

[37] T. Banks, M. Dine, and E. Gorbatov, "Is there a string theory landscape?," hep-th/0309170. 